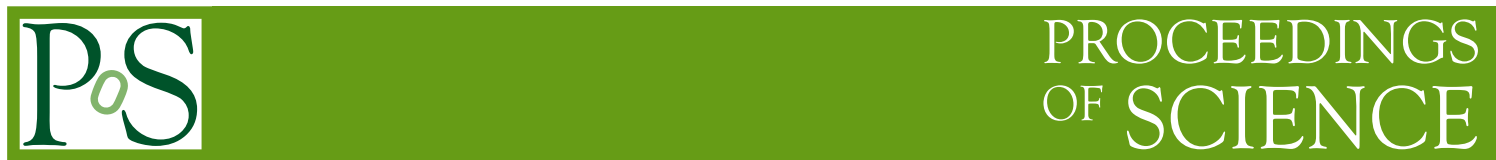

\title{
Recent results on correlations and fluctuations from lattice QCD
}

\author{
Szabolcs Borsanyi ${ }^{a}$, Zoltan Fodor ${ }^{a, b, c}$, Sandor D. Katz ${ }^{a, c}$, Stefan Krieg ${ }^{a, b}$, Claudia \\ Ratti $^{* d}$, Kalman K. Szabo ${ }^{a}$ \\ ${ }^{a}$ Bergische Universität Wuppertal, D-42119 Wuppertal, Germany \\ ${ }^{b}$ IAS, Jülich Supercomputing Centre, Forschungszentrum Jülich, D-52425 Jülich, Germany \\ ${ }^{c}$ Institute for Theoretical Physics, Eötvös University, H-1117 Budapest, Hungary \\ ${ }^{d}$ Università degli Studi di Torino and INFN, Sezione di Torino, I-10125 Torino, Italy \\ E-mail: ratti@to.infn.it
}

We summarize the new results of the Wuppertal-Budapest lattice QCD collaboration on flavor diagonal and non-diagonal quark number susceptibilities with $2+1$ staggered quark flavors, in a temperature range between 125 and $400 \mathrm{MeV}$. A Symanzik improved gauge and a stout-link improved staggered fermion action is utilized; the light and strange quark masses are set to their physical values. Lattices with $N_{t}=6,8,10,12,16$ are used. We perform a continuum extrapolation of all observables under study. All results are compared to the Hadron Resonance Gas model predictions: good agreement is found in the temperature region below the phase transition.

50th International Winter Meeting on Nuclear Physics - Bormio2012,

23-27 January 2012

Bormio, Italy

\footnotetext{
${ }^{*}$ Speaker.
} 


\section{Introduction}

A transition occurs in strongly interacting matter from a hadronic, confined system at small temperatures and densities to a phase dominated by colored degrees of freedom at large temperatures or densities. It was shown that the transition is merely an analytic crossover [1]. This field of physics is particularly appealing because the deconfined phase of QCD can be produced in the laboratory, in the ultrarelativistic heavy ion collision experiments at CERN SPS, RHIC at Brookhaven National Laboratory, ALICE at the LHC and the future FAIR at the GSI. A lot of effort is invested, both theoretically and experimentally, in order to find observables which would unambiguously signal the QCD phase transition. Correlations and fluctuations of conserved charges have been proposed long ago to this purpose $[2,3]$. The idea is that these quantum numbers have a very different value in a confined and deconfined system, and measuring them in the laboratory would allow to distinguish between the two phases.

Fluctuations of conserved charges can be obtained as linear combinations of diagonal and non-diagonal quark number susceptibilities, which can be calculated on the lattice at zero chemical potential $[4,5]$. These observables can give us an insight on the nature of the matter under study $[4,6]$. Diagonal susceptibilities measure the response of the quark number density to changes in the chemical potential, and show a rapid rise in the vicinity of the phase transition. Non-diagonal susceptibilities give us information about the correlation between different flavors. They are supposed to vanish in a non-interacting quark-gluon plasma (QGP). It was shown in Ref. [7] that correlations between different flavors are nonzero in perturbative QCD at large temperatures due to the presence of flavor-mixing diagrams: a quantitative analysis of this observable allows one to draw conclusions about the presence of bound states in the QGP [11]. Another observable which was proposed to this purpose, and which can be obtained from a combination of diagonal and non-diagonal quark number susceptibilities, is the baryon-strangeness correlator [12].

In the present contribution we show the results of our collaboration on some of these observables, with $2+1$ staggered quark flavors, in a temperature regime between 125 and $400 \mathrm{MeV}$ [13]. The light and strange quark masses are set to their physical values. Lattices with $N_{t}=6,8,10,12$ are used. Continuum extrapolations are performed for all observables under study. We compare our results to the predictions of the HRG model with resonances up to $2.5 \mathrm{GeV}$ mass at small temperatures, and of the Hard Thermal Loop (HTL) resummation scheme at large temperatures, when available. The details of the present analysis can be found in [13].

\section{Details of the lattice simulations}

The lattice action is the same as we used in $[14,15]$, namely a tree-level Symanzik improved gauge, and a stout-improved staggered fermionic action (see Ref. [16] for details). The stoutsmearing [17] yields an improvemed discretization of the fermion-gauge vertex and reduces a staggered artefact, the so-called taste violation (analogously to ours, an alternative link-smearing scheme, the HISQ action [18] suppresses the taste breaking in a similar way. The latter is used by the hotQCD collaboration in its latest studies $[19,20,21])$. Taste symmetry breaking is a discretization error which is important mainly at low energies. In the staggered fermion formulation, hadron masses cannot be uniquely determined at any finite lattice spacing [22]. Each continuum hadron 
state has a corresponding multiplet of states on the lattice: due to the taste symmetry violation the masses of these states are split. The impact of this effect on the thermodynamic observables has been recently discussed in the HRG framework $[23,10]$.

For details about the simulation algorithm we refer the reader to [15].

In analogy with what we did in $[14,15]$, we set the scale at the physical point by simulating at $T=0$ with physical quark masses [15] and reproducing the kaon and pion masses and the kaon decay constant. This gives an uncertainty of about $2 \%$ in the scale setting.

We used 2+1 SSavor QCD within the staggered framework, which needs taking the root of the fermion determinant. There is a lively discussion in the literature whether this is a correct procedure. Though we have not seen any problem with this fermion formalism (our results and the predictions of the hadron resonance gas model agree very nicely up to the transition region) it is still very important to repeat the calculations with actions, which are free of the rooting problem (e.g. Wilson fermions) [24].

\section{Observables under study}

The baryon number $B$, strangeness $S$ and electric charge $Q$ fluctuations can be obtained, at vanishing chemical potentials, from the QCD partition function. The relationships between the quark chemical potentials and those of the conserved charges are as follows:

$$
\begin{aligned}
\mu_{u} & =\frac{1}{3} \mu_{B}+\frac{2}{3} \mu_{Q} \\
\mu_{d} & =\frac{1}{3} \mu_{B}-\frac{1}{3} \mu_{Q} \\
\mu_{s} & =\frac{1}{3} \mu_{B}-\frac{1}{3} \mu_{Q}-\mu_{S} .
\end{aligned}
$$

Starting from the QCD pressure,

$$
\frac{p}{T^{4}}=\frac{1}{V T^{3}} \ln Z\left(V, T, \mu_{B}, \mu_{S}, \mu_{Q}\right)
$$

we can define the moments of charge fluctuations as follows:

$$
\chi_{\text {lmn }}^{B S Q}=\frac{\partial^{l+m+n} p / T^{4}}{\partial\left(\mu_{B} / T\right)^{l} \partial\left(\mu_{S} / T\right)^{m} \partial\left(\mu_{Q} / T\right)^{n}} .
$$

In the present paper we will concentrate on the quadratic fluctuations

$$
\chi_{2}^{X}=\frac{1}{V T^{3}}\left\langle N_{X}^{2}\right\rangle
$$

and on the correlators among different charges or quark flavors:

$$
\chi_{11}^{X Y}=\frac{1}{V T^{3}}\left\langle N_{X} N_{Y}\right\rangle .
$$

Given the relationships between chemical potentials (3.1) the diagonal susceptibilities of the conserved charges can be obtained from quark number susceptibilities in the following way:

$$
\chi_{2}^{B}=\frac{1}{9}\left[\chi_{2}^{u}+\chi_{2}^{d}+\chi_{2}^{s}+2 \chi_{11}^{u s}+2 \chi_{11}^{d s}+2 \chi_{11}^{u d}\right]
$$




$$
\begin{aligned}
\chi_{2}^{Q} & =\frac{1}{9}\left[4 \chi_{2}^{u}+\chi_{2}^{d}+\chi_{2}^{s}-4 \chi_{11}^{u s}+2 \chi_{11}^{d s}-4 \chi_{11}^{u d}\right] \\
\chi_{2}^{I} & =\frac{1}{4}\left[\chi_{2}^{u}+\chi_{2}^{d}-2 \chi_{11}^{u d}\right] \\
\chi_{2}^{S} & =\chi_{2}^{s} .
\end{aligned}
$$

If we do not wish to take further derivatives, we can take all three chemical potentials $(u, d, s)$ to zero. In this case, nothing distinguishes between the $u$ and $d$ derivative: this gives slightly simplified formulae:

$$
\begin{aligned}
& \chi_{2}^{B}=\frac{1}{9}\left[2 \chi_{2}^{u}+\chi_{2}^{s}+4 \chi_{11}^{u s}+2 \chi_{11}^{u d}\right], \\
& \chi_{2}^{Q}=\frac{1}{9}\left[5 \chi_{2}^{u}+\chi_{2}^{s}-2 \chi_{11}^{u s}-4 \chi_{11}^{u d}\right], \\
& \chi_{2}^{I}=\frac{1}{2}\left[\chi_{2}^{u}-\chi_{11}^{u d}\right] .
\end{aligned}
$$

The baryon-strangeness correlator, which was proposed in Ref. [12] as a diagnostic to understand the nature of the degrees of freedom in the QGP, has the following expression in terms of quark number susceptibilities:

$$
C_{B S}=-3 \frac{\left\langle N_{B} N_{S}\right\rangle}{\left\langle N_{S}^{2}\right\rangle}=1+\frac{\chi_{11}^{u s}+\chi_{11}^{d s}}{\chi_{2}^{s}}
$$

\section{Results}

The first observables we discuss are the diagonal light and strange quark number susceptibilities: their behavior as functions of the temperature is shown in the two panels of Fig. 1. The different symbols correspond to different values of $N_{t}$, from 8 to 16 . The red band is the continuum extrapolation, obtained from the unimproved data, not from the improved ones shown in the figure. The continuum extrapolation is performed through a parabolic fit in the variable $\left(1 / N_{t}\right)^{2}$, over five $N_{t}$ values from 6 to 16 . The band shows the spread of the results of other possible fits. The comparison between the improved data and the continuum bands in the figure shows the success of the improvement program throughout the entire temperature range. Both observables show a rapid rise in a certain temperature range, and reach approximately $90 \%$ of the ideal gas value at large temperatures. However, the temperature around which the susceptibilities rise is approximately 15-20 MeV larger for strange quarks than for light quarks. Besides, the light quark susceptibility shows a steeper rise with temperature, compared to the strange quark one. They approach each other at high temperatures. The pattern of temperature dependence is strongly related to the actual quark mass. The difference between the light and strange susceptibilities here with physical masses is more pronounced than in earlier works with not so light pions Ref. [25]. This is more evident in Figure 2: in the left panel we show the continuum extrapolation of both susceptibilities on the same plot. In the right panel we show the ratio $\chi_{s} / \chi_{u}$ : it reaches 1 only around $300 \mathrm{MeV}$, while for smaller temperatures it is $<1$. It is worth noticing that all these observables agree with the corresponding HRG model predictions for temperatures below the transition. 

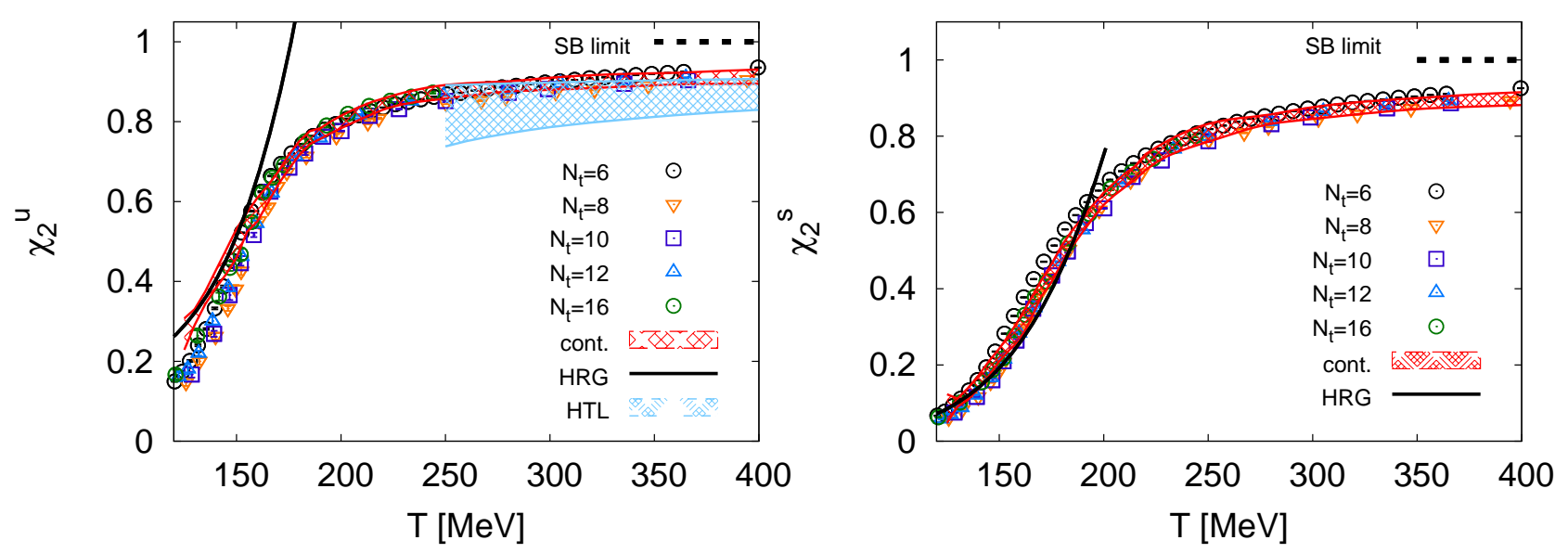

Figure 1: Left panel: diagonal light quark susceptibility as a function of the temperature. Right panel: diagonal strange quark susceptibility as a function of the temperature. In both panels, the different symbols correspond to different $N_{t}$ values. The red band is the continuum extrapolation. The black curve is the HRG model prediction for these observables. The dashed line shows the ideal gas limit. The light blue band in the left panel is the HTL prediction taken from Ref. [7].
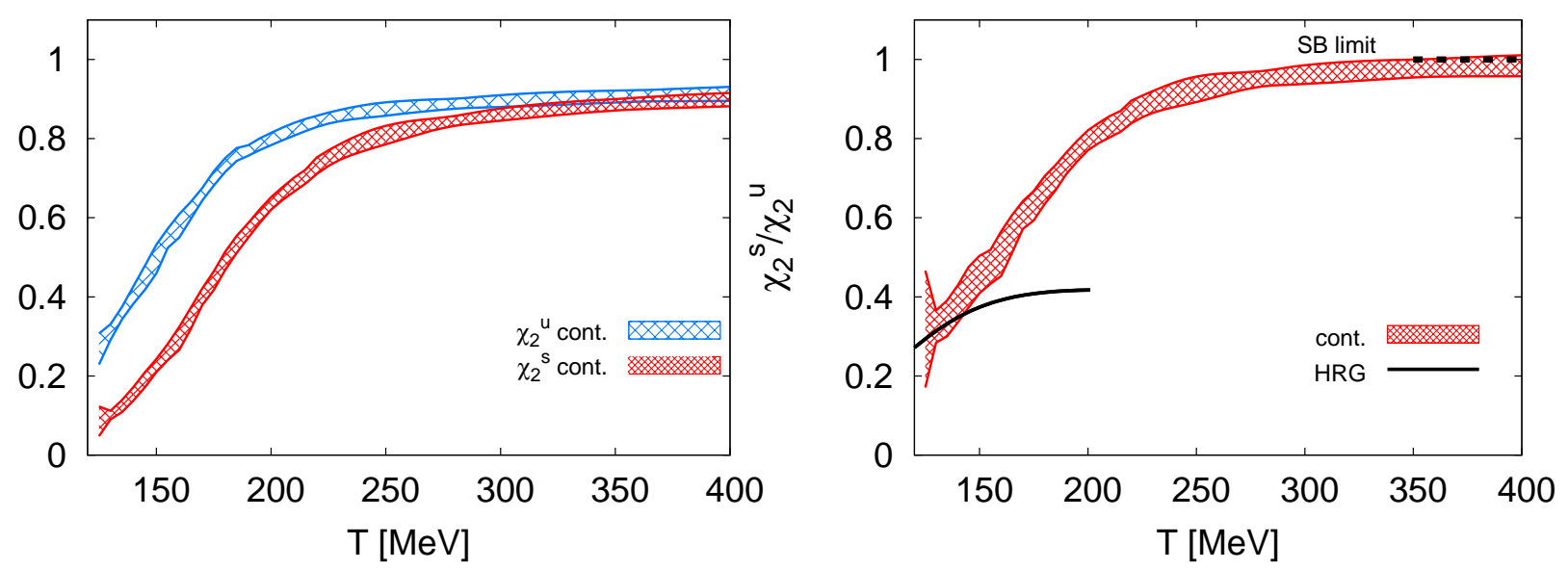

Figure 2: Left panel: direct comparison between the continuum limit of light and strange quark susceptibilities. Right panel: ratio $\chi_{2}^{s} / \chi_{2}^{u}$ as a function of the temperature. The red band is the lattice continuum result. The black, solid curve is the HRG model prediction. The dashed line indicates the ideal gas limit.

The non-diagonal us susceptibility measures the degree of correlation between different flavors. This observable vanishes in the limit of an ideal, non-interacting QGP. However, flavormixing diagrams in perturbative QCD yield a finite value for this correlation also at large temperatures [7]. We show our result in Fig. 3. $\chi_{11}^{u s}$ is non-zero in the entire temperature range under study. It has a dip in the vicinity of the transition, where the correlation between $u$ and $s$ quarks turns out to be maximal. It agrees with the HRG model prediction in the hadronic phase. This correlation stays finite and large for a certain temperature range above the transition temperature $T_{c}[8,9,10]$. A quantitative comparison between lattice results and predictions for a purely partonic QGP state can give us information about the probability of bound states survival above $T_{c}$ [11]. 


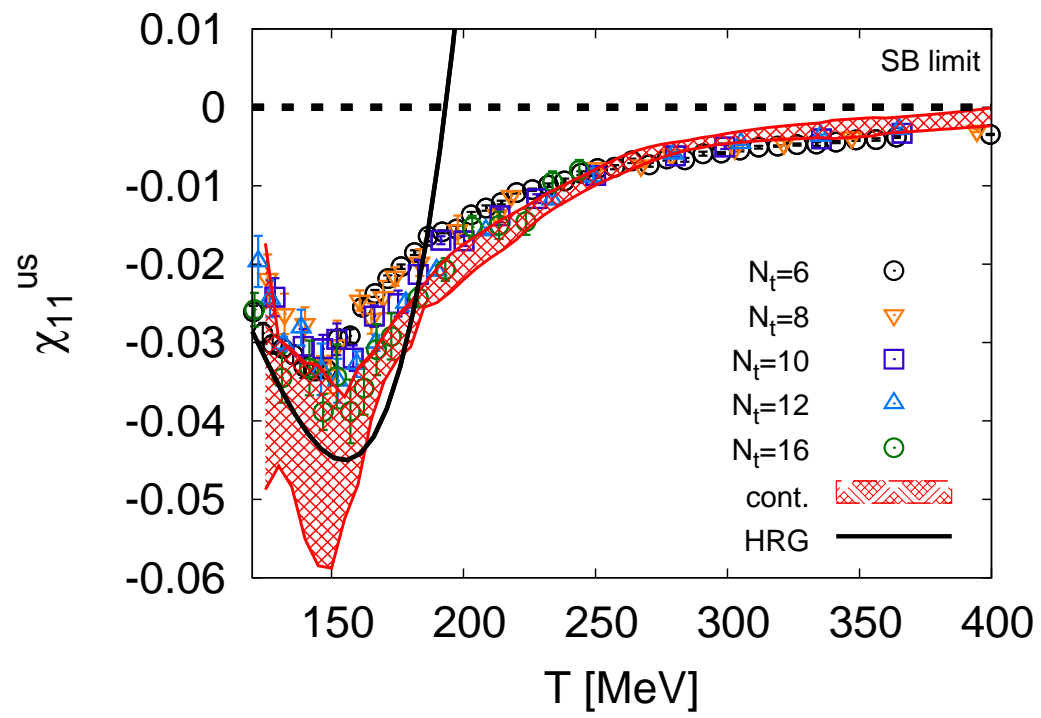

Figure 3: Non-diagonal u-s correlator as a function of the temperature. The different symbols correspond to different $N_{t}$ values. The red curve is the continuum extrapolated result. The black curve is the HRG model prediction. The dashed line indicates the ideal gas limit for this observable.

Quadratic baryon number, electric charge and isospin fluctuations can be obtained from the above partonic susceptibilities through Eqs. (3.7). We show our results for these observables in Fig. 4 and in the left panel of Fig. 5. In the low-temperature, hadronic phase we have a very good agreement with the HRG model predictions. In the vicinity of the phase transition, these quantities all show a rapid rise with temperature, in analogy with what already observed for the light and strange quark susceptibilities. At large temperature they reach approximately $90 \%$ of their respective ideal gas values. A comparison between all diagonal susceptibilities, rescaled by their corresponding Stefan-Boltzmann limits, is shown in the right panel of Fig. 5, from which it is evident that they all show similar features in their temperature dependence, even if the temperature at which they rise is larger for the strangeness and baryon number susceptibilities.

The baryon-strangeness correlator $C_{B S}$ defined in Eq. (3.8) was proposed long ago [12] as a diagnostic for strongly interacting matter. It is supposed to be equal to one for a non-interacting QGP, while it is temperature-dependent and generally smaller than one in a hadronic system. We show our result for this observable in Fig. 6. At the smallest temperatures it agrees with the HRG model result, and it shows a rapid rise across the phase transition. It reaches the ideal gas limit much faster than the other observables under study, yet there is a window of about $100 \mathrm{MeV}$ above $T_{c}$, where its value is still smaller than one. In analogy with $\chi_{11}^{u s}$, this observable seems to leave some room for bound states survival above $T_{c}$.

\section{Conclusions}

In this paper we have presented the continuum results of our collaboration on diagonal and non-diagonal quark number susceptibilities, in a system with $2+1$ staggered dynamical quark fla- 

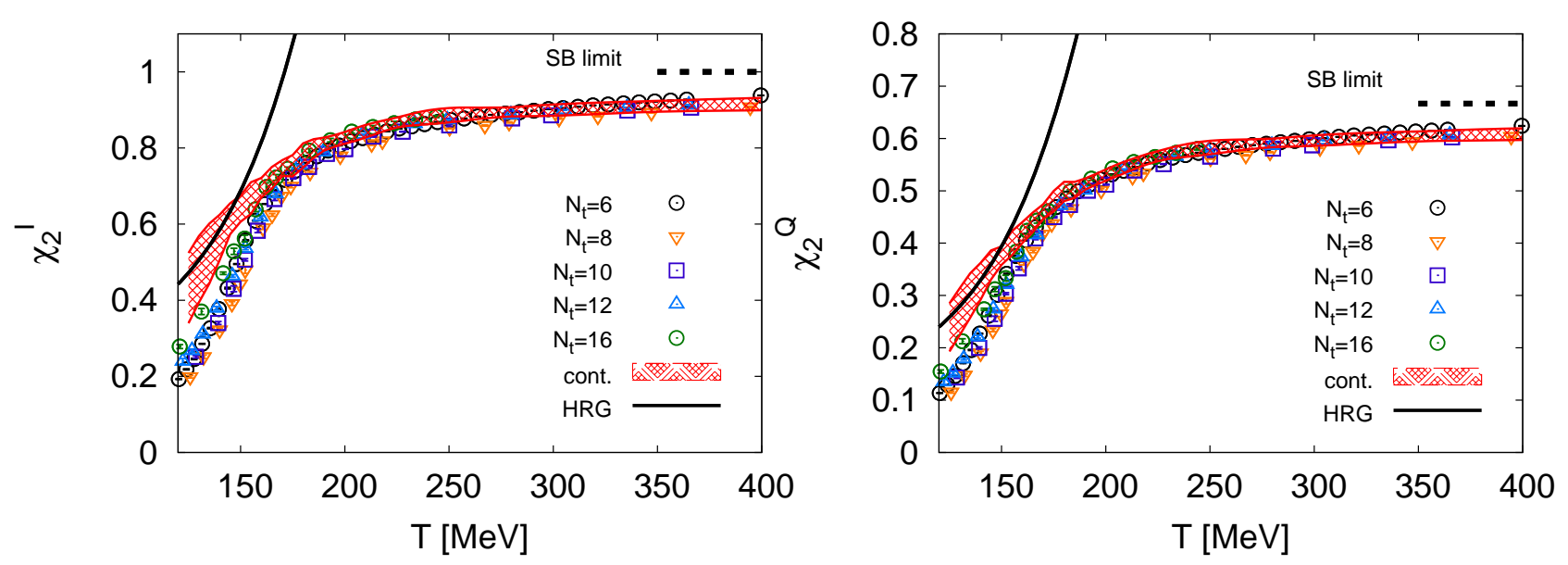

Figure 4: Left panel: isospin susceptibility as a function of the temperature. Right panel: electric charge susceptibility as a function of the temperature. In both panels, the different dots correspond to different $N_{t}$ values. The red band is the continuum extrapolation. The black curve is the HRG model prediction for these observables. The dashed line shows the ideal gas limit.
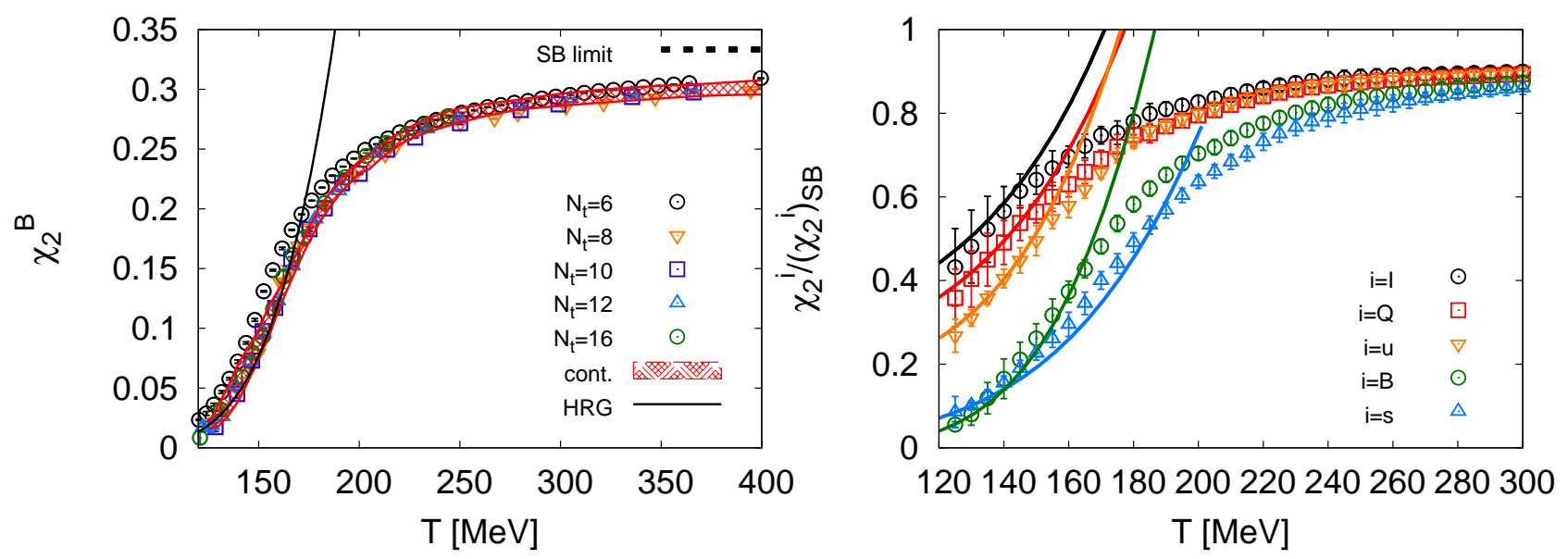

Figure 5: Left: quadratic fluctuation of baryon number as a function of the temperature. The different symbols correspond to different $N_{t}$ values, the red band is the continuum extrapolation and the black, solid curve is the HRG model result. The ideal gas limit is shown by the black, dashed line. Right: comparison between all diagonal susceptibilities, rescaled by the corresponding ideal gas limit, as functions of the temperature.

vors with physical masses, in a temperature range between 125 and $400 \mathrm{MeV}$. The continuum extrapolations were based on $N_{t}=6,8,10,12$ and 16 lattices. We calculated the systematic errors by varying over the ambiguities of the possible extrapolations.

All observables consistently show a very good agreement with the HRG model predictions for temperatures below the phase transition.

The diagonal fluctuations have some common features: they all show a rapid rise in the vicinity of the phase transition, and reach approximately $90 \%$ of the corresponding ideal gas value at large temperatures. The rise of both strange quark and baryon number susceptibilities is shifted to temperatures about $20 \mathrm{MeV}$ higher than those for light quark, charge and isospin susceptibilities. 


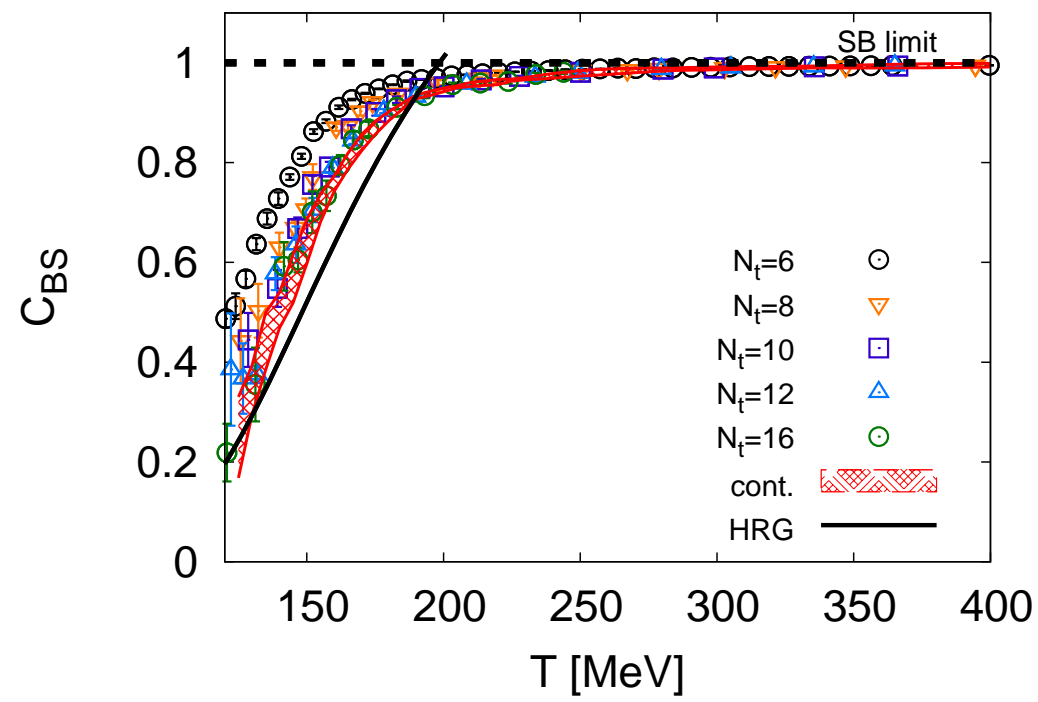

Figure 6: Baryon-strangeness correlator as a function of the temperature. The different symbols correspond to different $N_{t}$ values, the red band is the continuum extrapolation and the black, solid curve is the HRG model result. The ideal gas limit is shown by the black, dashed line.

Non-diagonal flavor and charge correlators remain different from their ideal gas values for a certain window of temperatures above the transition, thus not excluding the possibility of bound state survival above $T_{c}$.

\section{Acknowledgments}

Computations were carried out at the universities Wuppertal and Budapest on GPU clusters [26], on the Wuppertal QPACE machine and at Forschungszentrum Juelich. This work is supported in part by the Deutsche Forschungsgemeinschaft grants FO 502/2 and SFB- TR 55 and by the EU (FP7/2007-2013)/ERC no. 208740. The work of C. R. is supported by funds provided by the Italian Ministry of Education, Universities and Research under the Firb Research Grant RBFR0814TT.

\section{References}

[1] Y. Aoki, G. Endrodi, Z. Fodor, S. D. Katz and K. K. Szabo, Nature 443, 675 (2006) [hep-lat/0611014].

[2] S. Jeon and V. Koch, Phys. Rev. Lett. 85, 2076 (2000)

[3] M. Asakawa, U. W. Heinz and B. Muller, Phys. Rev. Lett. 85, 2072 (2000)

[4] S. A. Gottlieb, W. Liu, D. Toussaint, R. L. Renken, R. L. Sugar, Phys. Rev. Lett. 59, 2247 (1987). Phys. Rev. D38, 2888-2896 (1988).

[5] R. V. Gavai, J. Potvin, S. Sanielevici, Phys. Rev. D40, 2743 (1989).

[6] L. D. McLerran, Phys. Rev. D36, 3291 (1987).

[7] J. P. Blaizot, E. Iancu, A. Rebhan, Phys. Lett. B523, 143-150 (2001). [hep-ph/0110369]. 
[8] Y. Aoki, Z. Fodor, S. D. Katz and K. K. Szabo, Phys. Lett. B 643, 46 (2006) [hep-lat/0609068].

[9] Y. Aoki, S. Borsanyi, S. Durr, Z. Fodor, S. D. Katz, S. Krieg and K. K. Szabo, JHEP 0906, 088 (2009) [arXiv:0903.4155 [hep-lat]].

[10] S. Borsanyi et al. [Wuppertal-Budapest Collaboration], JHEP 1009, 073 (2010) [arXiv:1005.3508 [hep-lat]].

[11] C. Ratti, R. Bellwied, M. Cristoforetti, M. Barbaro, [arXiv:1109.6243 [hep-ph]].

[12] V. Koch, A. Majumder and J. Randrup, Phys. Rev. Lett. 95, 182301 (2005)

[13] S. Borsanyi, Z. Fodor, S. D. Katz, S. Krieg, C. Ratti and K. Szabo, JHEP 1201, 138 (2012) [arXiv:1112.4416 [hep-lat]].

[14] Y. Aoki, Z. Fodor, S. D. Katz and K. K. Szabo, Phys. Lett. B 643, 46 (2006)

[15] Y. Aoki, S. Borsanyi, S. Durr, Z. Fodor, S. Katz, S. Krieg and K. Szabo, JHEP 0906, 088 (2009)

[16] Y. Aoki, Z. Fodor, S. D. Katz and K. K. Szabo, JHEP 0601, 089 (2006)

[17] C. Morningstar and M. J. Peardon, Phys. Rev. D 69, 054501 (2004)

[18] E. Follana et al. [HPQCD and UKQCD Collaborations], Phys. Rev. D 75 (2007) 054502.

[19] A. Bazavov and P. Petreczky, PoS LAT2009, 163 (2009)

[20] A. Bazavov and P. Petreczky, arXiv:1005.1131 [hep-lat].

[21] A. Bazavov et al., arXiv:1111.1710 [hep-lat].

[22] N. Ishizuka, M. Fukugita, H. Mino, M. Okawa, A. Ukawa, Nucl. Phys. B411, 875-902 (1994).

[23] P. Huovinen, P. Petreczky, Nucl. Phys. A837, 26-53 (2010). [arXiv:0912.2541 [hep-ph]].

[24] S. Chandrasekharan, U.-J. Wiese Progress in Particle and Nuclear Physics 53, 373 (2004).

[25] R. V. Gavai, S. Gupta, Phys. Rev. D73, 014004 (2006). [hep-lat/0510044]. M. Cheng, P. Hendge, C. Jung, F. Karsch, O. Kaczmarek, E. Laermann, R. D. Mawhinney, C. Miao et al., Phys. Rev. D79, 074505 (2009). [arXiv:0811.1006 [hep-lat]]; A. Bazavov et al., Phys. Rev. D 80, 014504 (2009).

[26] G. I. Egri, Z. Fodor, C. Hoelbling, S. D. Katz, D. Nogradi and K. K. Szabo, Comput. Phys. Commun. 177, 631 (2007) [hep-lat/0611022]. 